\title{
Die „neue Linke“ als Außenzirkel der Macht. Rortys Herrschaftskritik und sein Weg aus der Theoriefalle
}

\begin{abstract}
„Bei ihrer Art zu reden bleibt der lebensnahe, den Kern der Probleme ansprechende Ton auf der Strecke. Anstatt zum Beispiel zu sagen, es sei ungeheuerlich, dass die Schulen in den Vorstädten so viel sicherer und sauberer sind als die Schulen in den Innenstädten, sagen sie, es sei uns nicht gelungen, die Andersheit des Anderen nicht in ausreichendem Maße anzuerkennen. Sie diagnostizieren dieses Scheitern als Symptom des allgemeinen Scheiterns der liberalen Demokratie - ein Symptom, das nur auf einem ziemlich hohen philosophischen Niveau verstanden werden kann“ (Rorty 2000a: 67).
\end{abstract}

\section{Einleitung}

Während es in der Philosophie eine weitreichende Debatte rund um das Werk Richard Rortys gibt, scheint der Autor in der deutschsprachigen Politikwissenschaft immer noch verhalten wahrgenommen zu werden. Dabei sind die wenigen existierenden politikwissenschaftlichen Auseinandersetzungen mit Rorty von zwei Schlagseiten geprägt. Auf der einen Seite betrachten Autoren wie Dirk Auer (2004), trotz grundlegender Sympathien, Rortys politische Theorie mit einiger Skepsis. Grund dafür ist, dass Auer in Rortys politischer Theorie einen Widerspruch zwischen dessen philosophischen und politischen Überlegungen erkennt (vgl. Auer 2004: 74). Nach Auer zeige sich dieser vor allem in Rortys mangelndem Verständnis des konflikthaften Wesens von Politik. Würde Rorty seinem philosophischen Anti-Essentialismus treu bleiben, so Auer, dann müsste er konsequenterweise die politische Gesellschaft als sozial unbestimmt und umkämpft beschreiben. Stattdessen vertrete Rorty jedoch ein „konsensualistisches Modell“ (ebd.), das nicht Konflikte, sondern die Betonung eines gemeinsamen politischen Vokabulars hervorhebe. Um diese theoretischen Inkonsistenzen zu beseitigen und Rortys Schriften für aktuelle politische Auseinandersetzungen anschlussfähig zu machen, fordert Auer, Rortys politische Theorie mit ,radikaleren' Theorien - etwa der Hegemonietheorie Laclaus und Mouffes - zusammen zu denken (vgl. ebd.: 132ff), also mit Ansätzen, denen Rorty explizit kritisch gegenüberstand (Rorty 1999c; 1999d). ${ }^{1}$

1 Dieser Vorschlag, Rortys politische Theorie anschlussfähiger zu machen, indem sie mit der ,radikalen Demokratietheorie‘ angereichert wird, findet sich jüngst auch bei Chin (2018). 
Auf der anderen Seite scheint eine liberale Position versucht, von vornherein jegliche Widersprüche aus dem Weg räumen zu wollen, indem sie Rorty in die Traditionslinie eines bürgerlich-liberalen Denkens stellt. So sieht Jens Hacke in ihm gar das US-amerikanische Pendant zur bundesrepublikanischen liberalkonservativen RitterSchule. In diesem Sinne würden Rortys Schriften der „defensiven Bürgerlichkeit“ eines Thomas Mann oder Odo Marquard gleichen (vgl. Hacke 2006: 274). Defensiv sei Rortys Bürgerlichkeit, weil der „,vormals mit den Linken sympathisierende“ Rorty die „bürgerlich-liberalen Tugenden und Werte vor allem durch deren Negation schätzen lernte" (ebd.). Rortys angeblicher Bruch mit der politischen Linken, den Hacke vor allem in der autobiographisch geprägten Schrift Stolz auf unser Land (Rorty 1999b) begründet sieht, ist für diese liberale Perspektive der Schlüssel für ein besseres Verständnis seiner Texte.

In diesem Beitrag wollen wir zeigen, dass beide Annäherungsversuche an Rortys politisches Denken zu kurz greifen. Im Gegensatz zur skeptischen Position wollen wir Rortys Werk nicht durch Theorien interpretieren, die er - wie im Falle radikaler Demokratietheorien - vehement kritisierte. Vielmehr gilt es, den vom Autor selbst gegebenen Hinweisen zu folgen, um Rortys Vermächtnis als politischer Intellektueller zu beleuchten. Dafür scheinen uns zwei Vorbilder Rortys besonders wichtig zu sein: John Dewey und George Orwell. Beide Autoren treten in Rortys Texten immer wieder auf und stellen zentrale Ankerpunkte seiner Überlegungen dar. Dennoch werden diese Wegweiser in der politikwissenschaftlichen Auseinandersetzung mit Rorty kaum beachtet. Diese Leerstelle gilt es zu füllen.

Im zweiten Abschnitt dieses Beitrags soll zunächst der Bedeutung John Deweys für Rortys politische Überzeugungen nachgegangen werden. Entgegen der liberalen Position argumentieren wir, dass Rorty sich dezidiert an einem demokratischen Sozialismus, so wie er von Dewey in den USA geprägt wurde, orientiert hat. Diese Parteinahme zeigt sich auch in Rortys Kritik an einer „,neuen Linken“. Wie wir im dritten Abschnitt zeigen wollen, hat sich diese „,neue Linke“ nach Rorty weitestgehend von den politischen Anliegen eines Großteils der Gesellschaft entfernt und so den Aufstieg des Rechtspopulismus mitbegünstigt. Vor dem Hintergrund eines derzeit breit diskutierten neuen kulturellen Klassenkonflikts in liberalen Demokratien soll dieser Kritik im vierten Abschnitt weiter nachgegangen werden. Dabei zeigt sich eine herrschaftskritische Stoßrichtung in Rortys politischem Denken, die bislang kaum berücksichtigt worden ist. Da diese Herrschaftskritik Rortys maßgeblich von den Arbeiten George Orwells beeinflusst ist, werden in diesem Abschnitt die Parallelen zu Orwells Darstellung der sozialistischen Intellektuellen im England der 1930er herausgestellt. Im letzten Abschnitt werden wir Rortys Bedeutung für aktuelle politikwissenschaftliche Überlegungen anhand seiner Forderung nach einer Linken, die wieder die Sprache der Nicht-Akademiker spricht, deutlich machen. 
Ohne Frage ist die verbreitete Rezeption Rortys als liberal-bürgerlicher Denker maßgeblich vom Autor mitverschuldet. Schließlich hat sich Rorty als postmoderner liberaler Bourgeois (vgl. Rorty 1991) oder auch als „bourgeoisen liberalen Marxisten“ (Rorty 2010: 21, Übers. d. A.) beschrieben. Diese durchaus überraschenden Selbstbezeichnungen zeigen jedoch, dass Rortys sie nicht ohne Selbstironie und durchaus als Seitenhieb gegen seine linken oder rechten Kritiker benutzte. Doch fällt im Zeitverlauf eine gewisse Radikalisierung seiner politischen Forderungen auf. Hat Rorty sich in Kontingenz, Ironie und Solidarität (2012 [1989]) und in seiner Auseinandersetzung mit Richard Bernstein (1987) deutlich von ,radikalen', marxistischen Positionen abgegrenzt, so findet sich in der zehn Jahre später erschienenen Essaysammlung Philosophy and Social Hope ein bemerkenswertes Zugeständnis gegenüber marxistischen Positionen: „Meines Erachtens hatten die Marxisten zumindest in einem Punkt Recht: Die zentralen politischen Fragen richten sich auf das Verhältnis zwischen Arm und Reich“ (Rorty 1999a: 232, Übers. d. A.). Zwar kommt es unmittelbar im Anschluss zu einer Distanzierung von der marxistischen Orthodoxie, doch insgesamt kommt in den Schriften aus den letzten Lebensjahren Rortys eine stärkere Sensibilisierung gegenüber sozialen Spaltungstendenzen, verbunden mit der Forderung nach einer Rückkehr zur Klassenpolitik, zum Vorschein. Zugleich beklagt Rorty den Verlust an Demokratie, der mit dem Globalisierungsprozess einhergeht. Charakteristisch ist etwa folgende Passage: „Wir haben jetzt eine globale Oberklasse, die alle wichtigen wirtschaftlichen Entscheidungen trifft und dies in völliger Unabhängigkeit von den Gesetzgebern und erst recht von dem Willen der Wähler irgendeines Landes“ (ebd.: 233; Übers. d. A.). Rorty schreibt diese Diagnose im Jahr 1996, also fast zehn Jahre vor der berühmten Post-Demokratie Diagnose von Colin Crouch (2005).

Doch Rorty bleibt nicht bei dieser Kritik stehen, sondern fordert - auch hier in Übereinstimmung mit Crouch - eine Rückbesinnung auf die Tradition der ,reformistischen Linken“. Unter dieser versteht er mit Blick auf die USA jene Gruppen, „die zwischen 1900 und 1964 im Rahmen der konstitutionellen Demokratie für den Schutz der Schwachen vor den Starken kämpften“ (Rorty 1999b: 45) und dann mehr und mehr von der „,neuen“ oder auch „kulturellen Linken“ verdrängt worden sind. Im Mittelpunkt dieser ,,reformistischen Linken“ stand, wie Rorty nicht müde wird zu betonen, die Forderung nach Umverteilung, der „Kampf für soziale Gerechtigkeit“ (ebd.: 53). ${ }^{2}$ Vor dem Hintergrund der Wiederkehr der Klassenspaltung in den USA seit den 1980er Jahren fordert Rorty eine Rückbesinnung auf die Tradition der reformistischen Linken, gerade auch, um sich der rechtspopulistischen Revolte entgegen-

2 Auf Rortys Kritik der „,neuen Linken“ beziehungsweise der Kulturlinken werden wir im nächsten Abschnitt noch ausführlicher eingehen. 
zustellen. In diesem Zusammenhang beruft er sich neben Herbert Croly insbesondere auf John Dewey. Es lohnt sich, diese Spur etwas weiter zu verfolgen.

Der Name Dewey wird von Rorty in seinen Schriften seit Der Spiegel der Natur (1987) ständig bemüht, etwa im Zusammenhang mit seiner Kritik der analytischen Philosophie, der konventionellen Wahrheitstheorie oder auch politiktheoretischen Fundierungsbemühungen. Nicht nur Dewey-Experten haben diese Vereinnahmung von Dewey kritisiert und darauf hingewiesen, dass Rortys Dewey wenig mit dem historischen Dewey zu tun habe. ${ }^{3}$ Auch Rorty selbst hat sich von Dewey's Metaphysics (Rorty 1982) und seinem Hegelianismus (Rorty 2000b) distanziert. Wie auch immer man die Frage der Übereinstimmung zwischen dem historischen und Rortys Dewey beurteilen mag, das Bild, das Rorty von Deweys politischen Engagement in Stolz auf unser Land zeichnet, ist zutreffend. Dewey war, wie auch Robert Westbrook (1991) aufgezeigt hat, ein „demokratischer Sozialist“, der sich zeitlebens für die Überwindung der Klassenspaltungen eingesetzt und sich politisch auf Seiten der „reformistischen Linken“ engagiert hat. Das gilt auch für die Jahre nach dem Ausbruch der Weltwirtschaftskrise, als er sich für radikale wirtschaftspolitische Maßnahmen ausgesprochen hat. Dazu zählte nicht zuletzt die Forderung nach einer deutlich progressiven Lohnsteuer, einer Verstaatlichung der Bodenschätze, der Schlüsselindustrien sowie der Banken. All diesen Forderungen lässt sich aus heutiger Sicht ganz gewiss ein sozialistischer und eben nicht liberaler Gehalt zuschreiben. Doch worauf es Rorty ankommt, ist weniger die Radikalität dieser Forderungen als der Umstand, dass Dewey diese im Rahmen der liberalen Demokratie, also vorwiegend mittels demokratischer Mehrheiten umzusetzen bestrebt war und sie als Experimente zur Lösung der „Probleme der Menschen“ (Rorty 1999b: 94)4 verstand. Und ganz ähnlich wie Rorty war Dewey, bei aller Nähe zu sozialistischen Forderungen, immer auch um Distanz zum orthodoxen Marxismus bemüht. ${ }^{5}$

Deweys Kritik war zunächst strategischer Natur. Er zeigte sich - durchaus zutreffend - davon überzeugt, dass in den USA sozialistische Theorien auf eine starke Ablehnung stoßen. Vor diesem Hintergrund betrieb er insbesondere in Individualism. Old and New und Liberalism and Social Action eine Neubeschreibung des Freiheitsbegriffs. Ausgangspunkt ist die Beobachtung, dass das Ideal der individuellen Freiheit für die meisten Bürger in einer kapitalistischen Massengesellschaft lediglich ein leeres Versprechen darstellt. Die amerikanische Gesellschaft sei in eine mächtige Elite und eine Masse von ohnmächtigen Bürgern gespalten, für viele von ihnen existieren die grundlegenden liberalen, aber auch demokratischen Versprechen nur auf dem Papier. Der Einfluss von Industrie und Kapital seien einfach zu groß und die

3 Etwa Westbrook (1991: 539-542).

4 Rorty bezieht sich mit dieser Formulierung auf einen einflussreichen Sammelband mit Texten von Dewey (1946).

5 Im Folgenden stützen wir uns auf Jörke (2020). 
Einflussmöglichkeiten, deren Macht durch die demokratische Willensbildung zu begrenzen, seien kaum vorhanden.

Laut Dewey ist dies das absolute Gegenteil von Individualität und Freiheit. Wenn der Einzelne seine lebensbildende Umwelt nicht mehr oder nur unzureichend gestalten kann, ist er nicht frei. Er besitzt nicht das, was Dewey als „effektive Freiheit“ (Dewey 1930, LW 5: 27, Übers. d. A.) bezeichnet. Hinter dieser Kritik steht sein Ideal des individuellen Wachstums als Kernprinzip einer demokratischen Gemeinschaft. Der Kerngedanke seiner Argumentation ist, dass in einer kapitalistischen Gesellschaft individuelles Wachstum oder Selbstverwirklichung für einen großen Teil der Bevölkerung nicht möglich ist.

Dewey wendet sich insbesondere gegen die ,religiöse Idealisierung [...] des Privateigentums“ (Dewey 1996 [1927]: 144f). Eigentumsrechte seien nur ein Instrument - und hierin zeigt sich Deweys pragmatistische Grundüberzeugung -, das überprüft werden muss, wenn es seine Ziele nicht mehr erreicht. Ende des 18. und Anfang des 19. Jahrhunderts dienten Recht und damit auch Eigentumsrechte den Vielen im Kampf gegen feudale Bevormundung. Heute würden sie dagegen lediglich die Privilegien der Reichen absichern. Das Hauptkriterium für eine gerechte Gesellschaft ist laut Dewey nicht, wie im liberalen Erbe, der Schutz des individuellen Eigentums, sondern die Möglichkeit des Wachstums für alle. Wenn absolute Eigentumsrechte gegen dieses Ziel arbeiten, sind sie nicht mehr „wahr“ und müssen durch bessere Konzepte ersetzt werden:

„Der frühere Liberalismus betrachtete das private und konkurrierende wirtschaftliche Handeln der Individuen als Mittel zum Zwecke des sozialen Wohlergehens. Wir müssen die Perspektive umdrehen und sehen, dass die sozialisierte Wirtschaft das Mittel zum Zwecke der freien individuellen Entwicklung ist“ (Dewey 1935, LW 11: 63, Übers. d. A.).

Zugleich richtet sich Dewey gegen die antidemokratischen Strukturen kommunistischer Systeme. Ist er in den 1920er Jahren den kommunistischen Experimenten in der Sowjetunion und in China durchaus noch mit Wohlwollen begegnet, so ändert sich seine Haltung in den 1930er Jahren zunehmend. ${ }^{6}$ Insbesondere in Freedom and Culture (1939, LW 12) kommt es zu einer scharfen Kritik der marxistischen Theorie und kommunistischen Praxis, doch bereits in den Jahren zuvor hat sich Dewey von der kommunistischen Kommandowirtschaft distanziert und dem Modell einer geplanten Gesellschaft das einer demokratisch planenden Gesellschaft entgegengestellt (vgl. Westbrook 1991: 456). Auch wenn seine entsprechenden Ideen nicht sonderlich konkret waren, so zielten sie doch insgesamt auf eine Demokratisierung der Expertise und eine möglichst breiten Einbeziehung der verschiedenen sozialen Klassen.

6 Auch Rorty konnte dem Kommunismus nichts abgewinnen; vgl. etwa Against Bosses, Against Oligarchies (2002). 
Schließlich, und mit diesem Aspekt zusammenhängend, lehnt Dewey auch die marxistische Theorie des Klassenkampfes entschieden ab. Sie - und auch in diesem Punkt folgt Rorty Dewey - ist für ihn Ausdruck eines Strebens nach Gewissheit, das sich nicht nur notwendig blind zeigt für die realen historischen und gesellschaftlichen Gegebenheiten, sondern darüber hinaus zu einer dogmatischen Trennung politischer Kämpfe führt (vgl. Dewey 1998 [1929]). Dem setzt Dewey das Modell einer Allianz der Arbeiterklasse und der ebenfalls infolge der großen Depression strauchelnden unteren Mittelklasse entgegen. So schreibt er in seinem programmatischen Essay The Need for a New Party:

„Der erste Appell der neuen Partei muss sich an die so genannte Mittelschicht richten: an Berufstätige, darunter natürlich auch Lehrer, den durchschnittlichen Einzelhandelskaufmann, den einigermaßen wohlhabenden Hausbesitzer, den ökonomisch unter Druck stehenden Angestellten, einschließlich seines weiblichen Gegenübers, und den Landwirt“ (Dewey 1931, LW 6: 171, Übers. d. A.).

Weiter führt er aus, dass eine neue politische Bewegung den erreichten Lebensstandard dieser Gruppen verteidigen und nach Möglichkeit erweitern solle, und das würde nur zusammen mit der Arbeiterklasse gelingen: „Letztendlich kann keine Bewegung erfolgreich sein, der es nicht gelang, die energische Unterstützung der Arbeiterklasse für sich zu gewinnen“ (ebd.: 172, Übers. d. A.).

Auch wenn Deweys Einsatz für eine dritte Partei und eine Demokratisierung wirtschaftlicher Prozesse letztlich gescheitert ist, steht er doch für die Idee der sozialen Demokratie, mit Rorty gesprochen, für eine „reformistische Linke“, die in den 1930er bis 1960er Jahren in den USA durchaus einige Teilerfolge feiern konnte und erheblich zur Verbesserung des Lebens für viele beigetragen hat. Nicht zuletzt wurde der erwirtschaftete Wohlstand während des New Deals breiter verteilt, gerade auch mit Blick auf afroamerikanische Bürgerinnen und Bürger, die von dem Ausbau des Sozialstaates und einer Stärkung der Rechte der Arbeiter und Arbeiterinnen profitierten (Kennedy 1999). Auch Johnsons sozialdemokratisches Reformprogramm Great Society konnte sich auf diese „reformistische Linke“ stützen und hat in der zweiten Hälfte der 1960er Jahre ebenfalls zur Verbesserung der Lebensbedingungen der ärmeren Klassen sowie zum Abbau von Rassenschranken beigetragen (Dallek 2005). Doch, wie im Folgenden gezeigt werden soll, kam es laut Rorty in der Auseinandersetzung über den Vietnamkrieg zum Zerfall der „,reformistischen Linken“, zur Etablierung einer „,neuen“, vorwiegend akademischen Linken und in der Konsequenz zum Aufstieg der populistischen Rechten. 


\section{Rortys Kritik an der ,,neuen Linken“}

Laut Rorty haben sich in der Zeit des Vietnamkriegs viele Linke, insbesondere Studenten an amerikanischen Universitäten, enttäuscht von ihrem Land abgewandt. Nicht nur wegen des Krieges, sondern auch angesichts des alltäglichen Leids vieler Afroamerikaner nahmen diese Studenten die USA eher als ein „Reich des Bösen“ (Rorty 1999b: 67) denn als eine vorbildhafte und verbesserungswürdige Demokratie wahr. Als politisch verlässlich erschienen ihnen weder Gewerkschaften noch sozialdemokratische Gesetzesinitiativen, sondern einzig die „militantesten Protestbewegungen der Afro-Amerikaner" (ebd.). Im Zuge dieser Absetzungsbewegung habe sich eine „,neue Linke“ geformt, die sich laut Rorty immer weniger mit der Habgier der Reichen und dem US-amerikanischen Wirtschaftssystem auseinandersetzte, den klassischen Themen der reformistischen Linken, sondern vor allem den Kampf gegen Sadismus, das heißt Kampagnen gegen die Erniedrigung und Ausgrenzung von religiösen, ethnischen oder sexuellen Minderheiten als ihr zentrales Anliegen formulierte. Daher bezeichnet Rorty die „neue Linke“ auch als eine kulturelle Linke. Dabei bestreitet er keineswegs, dass sie wichtige historische Erfolge feiern konnte und für die moralische Identität des modernen liberalen Amerikas außergewöhnliches geleistet habe (ebd.: 69). Allerdings ging dieser Fortschritt, der sich in einem stärkeren Bewusstsein für die Anerkennung von bisherigen Minderheiten zeigte, mit einer wachsenden Ungleichheit und der ökonomischen Unsicherheit der ärmeren Bevölkerungsschicht einher. Doch da sich die „,neue Linke“ für die sozio-ökonomische Lage eines Großteils der US-amerikanischen Gesellschaft nicht mehr interessierte, prophezeite Rorty bereits 1998, dass eine populistische Rechte schließlich diese Lücke nutzen und sich als eine Schutzmacht einer weitestgehend vergessenen und abgehängten weißen Mittel- und Unterschicht präsentieren wird:

„Während die Linke wegsah, kam die Verbürgerlichung des weißen Proletariats, die im Zweiten Weltkrieg begann und bis zum Ende des Vietnamkrieges fortschritt, zum Stillstand und begann sich umzukehren. In Amerika wird jetzt das Bürgertum proletarisiert, und das dürfte zu einer populistischen Revolte von unten führen, wie sie Buchanan anfachen möchte“ (1999b: 81).

Dass diese Prognose Wirklichkeit wurde, liegt für Rorty zu einem erheblichen Teil auch an dem Bruch innerhalb der Linken. Dabei ist es wichtig zu beachten, dass er nicht nur die Vernachlässigung der Klassenpolitik als Grund für das Erstarken des Rechtspopulismus sieht. Gleichbedeutend, und in der Auseinandersetzung mit Rorty bislang zu wenig beachtet, ist seine Kritik an dem selbstgenügsamen Auftreten dieser ,neuen Linken“, vor allem ihrer akademischen Stichwortgeber. Während sich die „reformistische Linke“, wie am Beispiel John Deweys gezeigt, noch für gesamtgesellschaftliche Reformen und sozialpolitische Gesetzesinitiativen interessierte, ziehen sich die Intellektuellen der „,neuen Linken“ laut Rorty auf 
„eine welthistorische Marsmenschen-Perspektive zurück und reden von der ,postmodernen Situation', den ,Widersprüchen des Spätkapitalismus' oder der ,heutigen amerikanischen Kultur', statt als Mitglieder einer bestimmten politischen Gemeinschaft zu sprechen, die eine Verantwortung für die anderen Mitglieder dieser Gemeinschaft tragen. Bei ihrer Art zu reden bleibt der lebensnahe, den Kern der Probleme ansprechende Ton auf der Strecke“ (Rorty 2000a: 67).

Diese Kritik Rortys an der Distanz der Intellektuellen zu den alltäglichen Problemen der meisten Menschen ist ein immer wieder auftauchendes Thema in seinen Texten. Bereits in seiner pointierten Abrechnung mit der analytischen Philosophie in seinem Frühwerk Der Spiegel der Natur formuliert Rorty grundlegende Zweifel gegenüber jeglicher Form philosophisch gewonnener Autoritätsansprüche (vgl. Rorty 1987: 414). In seinen späteren Texten kritisiert er dann insbesondere den philosophischen Gestus der Theoriesprache der ,neuen Linken“. Immer wieder vertritt Rorty vehement die Ansicht, dass politische Fragen ,in einfache und gewöhnliche Worte gefasst werden sollten - in Worte, die keine philosophische Zergliederung benötigen und keine philosophischen Voraussetzungen haben“ (Rorty 1999c: 45). Daher sind für ihn vor allem radikaldemokratische Denker suspekt, die, wie zum Beispiel Chantal Mouffe, davon überzeugt sind, dass ,,jede Fassung der demokratischen Politik [...] auch ein Verständnis des Wesens der Politik“ (Mouffe 1999: 24), also eine philosophische Fundierung, benötigt. Doch für Rorty hat genau diese

„Überbewertung der Philosophie [...] an den Universitäten von Amerika und Großbritannien (an welchen Derridas, Laclaus und Mouffes Bücher sehr stark rezipiert und bewundert werden) dazu beigetragen, eine mit sich selbst beschäftige akademische Linke hervorzubringen, die für die eigentliche politische Diskussion weitestgehend irrelevant geworden ist“" (Rorty 1999d: 155).

Solch deutliche Worte gegenüber den Arbeiten seiner eigenen Zunft haben Rorty sowohl von rechten als auch linken Kritikern den Ruf eines polemischen Nestbeschmutzers eingebracht, der sich vergeblich nach der verlorenen Zeit der reformistischen Linken samt ihrer engagierten politischen Intellektuellen sehne (vgl. Posner 2001: 340). Selbst freundlich gesinnte Auseinandersetzungen mit Rorty sind, wie bereits in der Einleitung geschrieben, skeptisch hinsichtlich seiner strikten Abneigung gegenüber politischen Diskussionen, die sich einer aus seiner Sicht metaphysischen und daher wenig produktiven Sprache bedienen. Wie wir nachfolgend zeigen, sind jedoch weder Skepsis noch Nostalgie-Vorwürfe angebracht.

\section{Die Akademikerklasse als Außenzirkel der Macht}

Folgt man dem Soziologen Andreas Reckwitz, dann verläuft der Klassenkonflikt innerhalb liberaler Demokratien nicht mehr entlang des Konflikts zwischen Kapital 
und Arbeit, sondern entlang kultureller Distinktionen. Der zentrale Akteur in dieser neuen kulturellen Klassenkonfiguration ist ihm zufolge eine seit den 1980er Jahren sich formierende neue Mittelklasse, die Reckwitz auch „Akademikerklasse“ nennt. Die Mitglieder dieser Klasse zeichnen sich durch ein hohes kulturelles Kapital von meist akademischen Bildungsabschlüssen aus und arbeiten im Feld der Wissens- und Kulturökonomie (vgl. Reckwitz 2018: 274). Im Gegensatz zu den anderen Klassen, Reckwitz nennt eine alte Mittelklasse, bestehend vorwiegend aus Facharbeitern, mittleren wie unteren Angestellten und nicht zuletzt aus der wachsenden Gruppe der Rentner sowie eine neuen Unterklasse, der prekär Beschäftigte, Arbeitslose und Sozialhilfeempfänger angehören, lege die Akademikerklasse zudem einen besonderen Wert auf einen (Kultur-)Kosmopolitismus, der gleichzeitig mit der Abwertung aller Provinzialität und traditioneller Werte einhergehe, die die „kosmopolitische Akademikerklasse vor allem in der Unterklasse (zumeist ihrer einheimischen, sesshaften Fraktion) und im ,kleinbürgerlich" scheinenden alten Mittelstand“ (ebd.: 302) ausmache. Die Unterscheidung zwischen Kosmopolitismus und Provinzialismus gerate so zum entscheidenden Konfliktfeld in liberalen Demokratien. Wobei die Dominanz der „Akademikerklasse“ und die damit einhergehende kulturelle Abwertung großer Teile der alten Mittel- und der neuen Unterklasse zu politischen Protestreaktionen wie dem Brexit oder dem anhaltenden Erfolg rechtspopulistischer Parteien geführt habe (ebd.: 369; vgl. Iversen/Soskice 2019: Kapitel 5).

Diese Beschreibung der Akademikerklasse und des neuen kulturellen Klassenkonflikts weist erstaunliche Parallelen zu der oben skizzierten Kritik Rortys an der neuen, akademischen Linken und ihrer Distanz zu den alltäglichen Problemen der Menschen auf. Auch Rorty hatte dabei die Entstehung einer Zweiklassengesellschaft, in der die gut ausgebildeten, international mobilen Fachleute einer provinziellen und sich vernachlässigt fühlenden alten Mittel- und Unterschicht gegenüberstehen, vor Augen. Wichtig ist allerdings zu sehen, dass Rorty an entscheidender Stelle über die Darstellungen von Reckwitz hinausweist. Die kulturellen Distinktionen, die Reckwitz hervorhebt, sind Rorty zufolge weniger Ausdruck eines gewandelten Kapitalismus als Resultat der steigenden sozio-ökonomischen Ungleichheit. Im Gegensatz zu Reckwitz nimmt Rorty damit eine materialistische Position ein, die ihn zu einer deutlich herrschaftskritischeren Perspektive führt. Denn seiner Meinung nach mache die Akademikerklasse, wenn vielleicht auch nicht intendiert, gemeinsame Sache mit der Klasse der Superreichen:

„Ganze Kompanien energischer junger Unternehmer füllen die Erste Klasse der interkontinentalen Düsenflugzeuge, während die hinteren Plätze mit beleibten Professoren wie mir beladen sind, die zu interdisziplinären Tagungen an schönen Orten ausschwärmen. Doch dieser neuentstandene kulturelle Kosmopolitismus ist auf das reichste Viertel der Amerikaner beschränkt. Der neue wirtschaftliche Kosmopolitismus lässt eine Zukunft ahnen, in der der Lebensstandard der übrigen drei Viertel ständig sinkt. Uns steht wahrscheinlich am Ende ein Amerika der erblichen sozialen Kasten bevor" (Rorty 1999b: 83). 
Dabei handelt es sich um eine Diagnose, die jüngst eindrucksvoll von Thomas Piketty (2020) empirisch fundiert wurde. Piketty zeigt mit Blick auf die USA - und im etwas geringeren Umfang auch für europäische Gesellschaften - nicht nur eine dramatisch wachsende soziale Spaltung sowie eine erhebliche Umkehrung der Wählerschichten sozialdemokratischer Parteien auf, sondern zeigt sich, ganz im Sinne von Rorty, auch davon überzeugt, dass es zu einer Interessenkoalition aus „brahmanischer Linke“ und „kaufmännischer Rechte“ gekommen sei:

„Die ,brahmanische Linke“ mag etwas mehr Steuern fordern als die ,kaufmännische Rechte', um Gymnasien, Elitehochschulen, Kunst- und Kulturinstitutionen zu finanzieren. Beide Lager vereint aber ihre große Nähe zum aktuellen Wirtschaftssystem und zum gegenwärtigen Stand der Globalisierung. Der Status quo verschafft den intellektuellen und wirtschaftlichen Eliten ausreichend Vorteile“ (Piketty 2020: 957; vgl. ebd.: 1027).

Die Pointe oder auch Provokation von Pikettys Argumentation liegt nun darin, dass er in dieser Interessenkoalition eine „Rückkehr zur Grundlogik der alten trifunktionalen Gesellschaften, der Rollenverteilung zwischen intellektuellen und kriegerischen Eliten“ (ebd.: 957) erblickt. ${ }^{7}$ Diese Diagnose, gerade auch in ihrer polemischen Zuspitzung, wäre sicherlich auf Rortys Zustimmung getroffen. Um an dem wirtschaftlichen Kosmopolitismus teilzuhaben und auf den Hinterbänken der Wirtschaftselite auf Steuerzahlerkosten zu interdisziplinären Tagungen in der ganzen Welt zu fliegen, muss die „Akademikerklasse“ nämlich auch laut Rorty den Wirtschaftsbossen den Rücken frei halten, genauso, wie die intellektuellen und überwiegend religiösen Eliten in vergangenen Zeiten die Legitimität stratifikatorischer und feudaler Gesellschaftsordnungen abgesichert haben. In Anlehnung an George Orwells Dystopie Nineteen Eighty-Four spitzt Rorty das Bild einer vom Rest der Gesellschaft sprichwörtlich abgehobenen Herrschaftsklasse, in der es eine klare Aufgabenverteilung zwischen dem inneren Zirkel der Macht, den internationalen Superreichen und ihrem Außenzirkel, den kosmopolitischen akademischen Fachleuten, gibt, jedoch noch weiter zu:

„Wenn die Bildung erblicher Kasten unbehindert weitergeht und der Globalisierungsdruck solche Kasten nicht nur in den Vereinigten Staaten, sondern auch in den anderen alten Demokratien erzeugt, landen wir bei einer Orwellschen Welt. Vielleicht gibt es dort keinen übernationalen Großen Bruder und keine offizielle Ideologie wie Ingsoc. Aber es wird eine Parallele zur ,inneren Partei' geben - nämlich die internationalen kosmopolitischen Superreichen. Sie werden alle wichtigen Entscheidungen treffen. Die Parallele zu Orwells ,äußerer Partei` sind dann die gut ausgebildeten und gut gestellten kosmopolitischen Fachleute" (Rorty 1999b: 85).

Die Hauptfunktion dieser äußeren Partei ist es nach Rorty, die „Proletarier“ (ebd.) stillzuhalten. Das tut sie am besten, indem sie von den bestehenden Ungleichheiten

7 Ähnlich auch Kotkin (2020). 
ablenkt. Statt also über wirtschaftliche Entscheidungen zu diskutieren, die weiterhin die Sache der Superreichen sein sollen und von denen die äußere Partei beispielsweise in der Frage der steuerrechtlichen Begünstigung von Fernreisen zu schönen Tagungsorten in der ganzen Welt profitieren, spezialisieren sich politische Intellektuelle auf kulturelle oder philosophische Fragen, die für die eigentliche politische Diskussion weitestgehend irrelevant sind. Der entscheidende Punkt, den Rorty uns durch seinen Hinweis auf Orwell bietet, ist nun, dass die Akademikerklasse nicht nur eine - wie gesagt größtenteils nicht-intendierte - systemstabilisierende Funktion innehat, sondern auch, dass sie die Rolle als Steigbügelhalter der Superreichen bisweilen zu genießen scheint.

Auf diesen Punkt macht Rorty bereits zehn Jahre vor seiner hier skizzierten Kritik an der ,neuen Linken“ in seiner Interpretation des Romans Nineteen Eighty-Four in seinem Buch Kontingenz, Ironie und Solidarität (2012 [1989]) aufmerksam. Im Gegensatz zu vielen anderen versteht Rorty Orwells Dystopie nicht nur als eine Abrechnung mit totalitären Herrschaftssystemen, insbesondere der damaligen Sowjetunion. Vielmehr wechselt in Rortys Lesart Orwell im letzten Kapitel des Buches die Perspektive. In diesem Kapitel foltert ein Mitglied der äußeren Partei, der Parteiintellektuelle O’Brien, die Hauptfigur Winston, einen Mittelschichtangehörigen und Gegner des totalitären Systems, so lange bis dieser bereit ist, den Doktrinen der herrschenden Partei zu folgen und seine persönlichen Überzeugungen vollkommen aufzugeben. Nach Rorty habe Orwell mit dieser Folterszene nicht die grausamen Praktiken der damaligen Sowjetunion beschrieben, sondern eine Vision eines ,post-totalitären Staates“ entworfen, wobei Orwell der erste war, „der sich fragte, welches Selbstverständnis Intellektuelle in solchen Staaten haben würden, wenn erst einmal klar wäre, dass liberale Ideale sich nicht auf die Zukunft der Menschen beziehen. Die Antwort auf seine Frage heißt O'Brien“ (Rorty 2012 [1989]: 277). O’Brien symbolisiert für Rorty ein durchaus realistisches Szenario: Wenn linke Intellektuelle sich zunehmend vom Rest der Gesellschaft entfernen und jegliche Hoffnung auf eine Reform des bestehenden Gemeinwesens aufgeben, dann ist die einzige intellektuelle Tätigkeit, die ihnen als Mitglieder der äußeren Partei noch Genuss verschafft, ihre Rolle als Steigbügelhalter der Superreichen. In dieser Funktion müssen sie vor allem diejenigen Gesellschaftsmitglieder, die wie Winston noch Hoffnungen ${ }^{8}$ auf Veränderungen haben, kontrollieren und sie von ihren Überzeugungen abbringen.

In dieser frühen Kritik Rortys an der herrschaftsstabilisierenden Funktion linker Intellektueller bleibt zunächst noch unklar, inwiefern dieses drastische Bild Orwells für Rorty nur als Warnung verstanden werden muss oder seiner Meinung nach auf große Teile der akademischen Linken zutrifft. Rorty selbst gibt in diesem Abschnitt

8 Im Roman verortet Winston diese Hoffnung im Proletariat: „If there ist hope, it lies in the proles“ (Orwell 1987 [1949]: 64). Viele Orwell-Interpretationen sehen diese Hoffnung auch als entscheidend für Orwells politische und literarische Aktivitäten an (vgl. Newsinger 2018). 
zudem keine Hinweise darauf, welche Vorteile linke Intellektuelle von dieser Rolle haben - außer der dystopischen Darstellung O'Briens, der sich an der Demütigung seines Opfers berauscht. Um diesen Fragen nachzugehen, ist es daher sinnvoll, dem von Rorty selbst gegebenen Hinweis auf George Orwell zu folgen. Denn dieser skizziert nicht nur in seinen fiktiven Romanen Nineteen Eighty-Four (1949) und Animal Farm (1945) das düstere Bild einer abgehobenen und selbstzufriedenen intellektuellen Elite, sondern wirft bereits in seinem Werk Road to Wigan Pier (1937) einen kritischen Blick auf die sozialistischen Intellektuellen Englands. In diesem Buch schildert Orwell seine Erfahrungen, die er während einer Reise im Jahre 1936 durch die nordenglischen Berg- und Kohlebaugebiete machte. Nachdem er von dem harten Arbeitsalltag und den miserablen Lebensbedingungen der dort lebenden Menschen berichtet, wirft Orwell im letzten Kapitel die Frage auf, warum die Idee des Sozialismus in diesen Regionen nicht mehr Unterstützung findet. Angesichts der wachsenden sozialen Ungleichheit sei schließlich ,jeder leere Magen ein Argument für den Sozialismus“ (Orwell 1982: 167). ${ }^{9}$ Den Grund für diese fehlende Unterstützung macht Orwell schließlich im Verhalten der sozialistischen Intellektuellen ausfindig. Viele Arbeiter, so Orwell, werden nicht durch die sozialistischen Ziele, sondern durch die selbsternannten Fürsprecher des Sozialismus abgeschreckt. Da diese in der Mehrzahl aus der oberen Mittelklasse stammen, gleiche ihr Bekenntnis zum Sozialismus eher einer Lifestyle-Entscheidung und entspringe nicht aus dem Wunsch nach besseren Arbeitsbedingungen oder einer besseren finanziellen Lage. Zwar hätten die meisten „Mittelstandssozialisten“ (ebd.: 170) ein theoretisches Verständnis für die Anliegen der Arbeiter, in Wirklichkeit würden sie jedoch wie „Leim an den elenden Überresten ihres sozialen Prestiges kleben“ und „mit zugehaltener Nase“ fliehen, wenn sie auf einen ,noch von der Grube schmutzigen Bergmann“ treffen (ebd.: 171). Der typische Sozialist sei in England kein ,grimmig aussehender Arbeiter“ (ebd.: 169), sondern

„entweder ein jugendlicher bolschewistischer Snob, der in fünf Jahren wahrscheinlich eine gute Partie gemacht hat und zum römisch-katholischen Glauben übergetreten ist, oder, noch typischer, ein zimperlicher kleiner Mann mit einem Weißen-Kragen-Beruf, gewöhnlich heimlicher Abstinenzler und oft mit vegetarischen Neigungen, mit einer Geschichte des Nonkonformismus hinter sich, und vor allem, mit einer sozialen Stellung, die er keineswegs aufs Spiel setzen will“" (ebd.; Herv. d. A.).

Aufgrund solcher Beschreibungen lassen viele (neo-)marxistische Intellektuelle an Orwell - ganz ähnlich wie an Rorty - kaum ein gutes Haar (vgl. Norris 1984). Orwells entscheidender Kritikpunkt wird dabei jedoch nur selten berücksichtigt. Denn wie auch bei Rorty zu lesen, ist für Orwell die Verteidigung des sozialen Prestiges für das Verhalten der Mittelstandssozialisten entscheidend. Sie führen ein fi-

9 Wir zitieren im Folgenden aus der deutschsprachigen Übersetzung (vgl. Orwell 1982). 
nanziell gut gestelltes und zufriedenes Leben, das sie auf keinen Fall aufgeben möchten. ${ }^{10}$ Im Gegenteil, sozialistische Parteien und Gewerkschaften, so geht Orwells Kritik weiter, scheinen für sie ein willkommenes Terrain zu sein, um Karriere zu machen und ihr kulturelles Kapital zu demonstrieren. Dieses Distinktionsgehabe der selbsternannten Sozialisten lasse sich vor allem an einem abgehobenen Theoriejargon beobachten. Während Sozialismus für den ,gewöhnlichen Arbeiter, wie man ihn Samstagnacht in jeder Kneipe antrifft“, nicht viel mehr als „bessere Löhne und kürzere Schichten und niemand, der dich herumkommandiert" (Orwell 1982: 172) bedeute, gehe es für den „bolschewistischen Snob“ in erster Linie um die Erörterung der philosophischen Aspekte des Marxismus. Da die Akademiker in sozialistischen Parteien und Gewerkschaften tonangebend sind, ist es für Orwell nicht weiter überraschend, dass sich ein ,gewöhnlich anständiger Mensch“ (ebd.: 178) dort nicht mehr wohl fühlt und die Idee des Sozialismus damit gerade bei den hart arbeitenden Menschen an Attraktivität verliert. Angesichts dieser desolaten Situation stellt sich für Orwell die Frage, warum sich die hier beschriebenen sozialistischen Intellektuellen überhaupt für die Angelegenheiten der Arbeiter engagieren:

„Manchmal schaue ich einen Sozialisten an - den intellektuellen, Traktate verfassenden Typ, mit seinem Pullover, dem wirren Haar und den Marx-Zitaten - und frage mich, was zum Teufel wirklich sein Motiv ist. Oft fällt es schwer, an seine Liebe zu irgendjemandem zu glauben, besonders zu den Arbeitern, von denen er am allerweitesten entfernt ist. Das Motiv vieler Sozialisten ist meiner Meinung nach lediglich ein überentwickelter Ordnungssinn. Der gegenwärtige Zustand verletzt sie nicht deshalb, weil er Elend verursacht, und noch weniger, weil er Freiheit verunmöglicht, sondern weil er unordentlich ist; sie verlangen im Grunde danach, die Welt auf etwas wie ein Schachbrett zu reduzieren“ (ebd.: 174).

Für Richard Rorty ist dieses Bild eines empathielosen, nach Ordnung und philosophischer Reinheit strebenden linken Intellektuellen nach wie vor zutreffend. Die Rolle des orthodoxen Marxisten haben seiner Meinung nach nun „radikale“ Intellektuelle übernommen, die sich in „theoretischen Sophistereien“ verlieren und sich so verhalten, ,als seien praktische Szenarien unnötig, als könnten die Intellektuellen ihrer politischen Verantwortung einfach dadurch nachkommen, dass sie offensichtliche Missstände in der Terminologie immer ,radikalerer' theoretischer Vokabulare kritisieren“ (Rorty 2012 [1989]: 295, FN 15).

Ein Blick auf aktuelle Debatten nicht nur der deutschsprachigen Sozial- und Geisteswissenschaft scheint Rorty in dieser Bestandsaufnahme nach wie vor Recht zu geben. ${ }^{11}$ Von praktischen Vorschlägen zur Verbesserung der Lebensbedingungen der

10 Dieser Aspekt wird mit Blick auf den ökologischen Fußabdruck auch von Ingolfur Blühdorn et al. (2020) betont.

11 Auch wenn Rorty in politischen Angelegenheiten den radikalen Theorien der „neuen Linken“ nichts abgewinnen konnte, hob er doch auch immer wieder hervor, dass diese Theorien für einen privaten, ästhetischen Gebrauch durchaus wertvoll sein können (vgl. Rorty 2012 [1989]). 
Armen und der unter Druck geratenen unteren Mittelklasse oder dem Versuch, den Machenschaften der Reichen auf die Spur zu kommen, ist nur selten zu lesen. Stattdessen geht es um mehr oder weniger freundschaftlich ausgetragene akademische Gefechte - etwa um die Frage, mit welchem theoretischen Konstrukt der Gegenwartskapitalismus beschrieben, kritisiert und theoretisch überwunden werden kann, ob für den Aufstieg des Rechtspopulismus materielle oder kulturellen Faktoren entscheidend sind, oder auch, worin die philosophischen Fundamente der Gesellschaftskritik bestehen. ${ }^{12}$ Durch den Vergleich zu Orwell wird die herrschaftskritische Stoßrichtung in Rortys Darstellung dieser selbstreferentiellen „neuen Linken“ deutlich. Demnach geht es der akademischen Linken also überhaupt nicht um die Lösung aktueller politischer Probleme, wobei Rorty hier vor allem an sozialpolitische Fragestellungen dachte, sondern eher um das snobistische Wetteifern über ihre korrekte, vielmals philosophisch fundierte Beschreibung. Doch genau dadurch werden die realen Probleme und Sorgen eines Großteils der Gesellschaft zu akademisch-intellektuellen Erörterungen umgedeutet. Auf diesem Wege erfüllt die „Akademikerklasse“ ihre Rolle als Außenzirkel der Macht und sichert sich ihre Teilhabe am wirtschaftlichen Kosmopolitismus.

Rorty hat auf diese Entwicklung mit der Forderung nach einer Rückkehr zur Klassenpolitik und insbesondere einer Rückbesinnung auf die Programmatik der „reformistischen Linken“ reagiert (vgl. Rorty 1999a: 255ff). Wie in Orwells Darstellung deutlich werden sollte, ist damit jedoch nicht eine philosophische Debatte über die Grundlagen des Marxismus gemeint. Vielmehr geht es Rorty vor allem um eine Rückbesinnung auf die tatsächlichen Probleme der Menschen und der Überwindung der aktuell vorherrschenden Interessenkoalition aus „brahmanischer Linken“ und „kaufmännischer Rechten“.

\section{Rortys Forderung nach einer Rückbesinnung auf die Politik und Werte der alten Linken}

Viele Formulierungen von Rorty und im stärkeren Umfang von Orwell sind rhetorisch überspitzt. Nichtsdestotrotz wohnt ihnen ein wahrer Kern inne. Dieser besteht in der wachsenden Kluft zwischen ,linken' Funktionseliten und deren intellektuellen Stichwortgebern und großen Teilen der Bevölkerung. Wie vielfach empirisch belegt, haben gerade die ehemaligen Stammwähler sozialdemokratischer und links von diesen stehenden Parteien sich von diesen größtenteils verabschiedet. Sie sind entweder ins Nichtwählerlager gewandert oder machen, wie bereits erwähnt, ihr Kreuz bei rechtspopulistischen Parteien. ,Linke` Parteien werden gegenwärtig vorwiegend von

12 Die Beispiele sind zu zahlreich, als dass es sich lohnen würde, hier welche exemplarisch anzuführen. Einen schönen Überblick bietet Paoli (2017). 
Menschen mit höheren Bildungsabschlüssen, also in den Worten von Reckwitz, von den Angehörigen der „Akademikerklasse“ gewählt (vgl. Piketty 2020: 899-1084). Ganz offensichtlich vermögen es diese Parteien immer weniger, diejenigen zu erreichen, für deren Emanzipation sie zumindest auch zu kämpfen behaupten. Im Anschluss an Rorty und Orwell lässt sich verstehen, weshalb das der Fall ist. Sozialdemokratische und andere linke Parteien haben sich in den vergangenen Jahren stark akademisiert. Ein Prozess, der zwar auf nahezu alle politischen Parteien zutrifft und als Folge einer „Professionalisierung der Politik“ (Borchert 2003) einerseits und die Entwicklung hin zu Kartellparteien (Katz/Mair 1995) andererseits zu deuten ist. Zugleich stellt das für linke Parteien ein besonderes Problem dar. Denn in der Folge der Akademisierung ihres Personals ist eine lebensweltliche Kluft zu den Angehörigen der alten Mittelklasse und der neuen Unterklasse entstanden. ${ }^{13}$ Diese Kluft zeigt sich im Habitus, im Lebensstil, in der Sprache und nicht zuletzt in den Werten, die jeweils vertreten werden, wobei linke Parteien in all diesen Dimensionen sich mehr und mehr der Akademikerklasse angeglichen haben. Wie Nancy Fraser gezeigt hat, ist dabei ein „progressiver Neoliberalismus“ (Fraser 2017: 78) entstanden, der sich etwa in der Forderung nach Erhöhung des Frauenanteils in Spitzenunternehmen oder auch in den Bemühungen, in den oberen Etagen von Wirtschaft, Politik und Kultur die Diversität zu erhöhen, zeigt.

Rorty hat schon vor mehr als 20 Jahren vor dieser Entwicklung gewarnt. Zwar hat er, wie erwähnt, die Errungenschaften der „,neuen Linken“ im Kampf gegen den alltäglichen Sadismus, also den Kampf gegen die vielfältigen Diskriminierungen derjenigen, die nicht zur Mehrheitsgesellschaft gehören, explizit hervorgehoben als etwas, hinter den man nicht zurückfallen dürfe. Doch zugleich hat er gesehen, dass die Protagonisten dieser „,neuen Linken“ sich zu sehr von der Welt der unteren Klassen entfernt haben, und er dachte dabei eben nicht nur an die Angehörigen der weißen Arbeiterklasse. Gerade in den USA sind es nicht zuletzt Afroamerikaner und die Angehörigen ethnischer Minderheiten, die die rapide wachsenden Unterklassen ausmachen. ${ }^{14}$ Völlig zu Recht hat er darauf verwiesen, dass diese Klassen von der ,neuen Linken" nicht mehr erreicht würden und dass dafür nicht zuletzt deren Sprache und deren philosophischer Jargon verantwortlich seien. Rortys auch heute noch überzeugender Appell an die Linke bestand darin, sich wieder stärker den konkreten Problems of Men (Dewey 1946) zu widmen und sich dabei auch einer verständlicheren Sprache zu bedienen.

13 Mit Blick auf linke Parteien und Bewegungen in Deutschland vgl. Baron (2016).

14 Für eine ähnliche Argumentation mit Blick auf Deutschland vgl. Brinkmann/Jörke/Paulitz (2018). 
Auer, Dirk 2004: Politisierte Demokratie. Richard Rortys politischer Antiessentialismus. Wiesbaden.

Baron, Christian 2016: Proleten, Pöbel, Parasiten. Warum die Linken die Arbeiter verachten. Berlin.

Bernstein, Richard 1987: „One Step Forward, Two Steps Backward. Richard Rorty on Liberal Democracy and Philosophy“. In: Political Theory, 15 (4), S. 538-563.

Blühdorn, Ingolfur et al. 2020: Nachhaltige Nicht-Nachhaltigkeit. Warum die ökologische Transformation der Gesellschaft nicht stattfindet. Bielefeld.

Borchert, Jens 2003: Die Professionalisierung der Politik. Zur Notwendigkeit eines Ärgernisses. Frankfurt/New York.

Brinkmann, Ulrich/Jörke, Dirk/Paulitz, Tanja 2018: „Raus aus der neoliberalen Umarmung“. In: Forum Wissenschaft, 4, S. 49-52.

Chin, Clayton 2018: The Practice of Political Theory. Rorty and Contintental Thought. New York.

Crouch, Colin 2005: Post-Democracy. Oxford.

Dallek, Robert 2005: Lyndon B. Johnson. Portrait of a President. Oxford.

Dewey, John 1946: The Problems of Men. New York.

Dewey, John 1996 [1927]: Die Öffentlichkeit und ihre Probleme. Bodenheim.

Dewey, John 1998 [1929]: Die Suche nach Gewißheit. Eine Untersuchung des Verhältnisses von Erkenntnis und Handeln. Frankfurt.

Dewey, John: The Later Works (1925-1952), 17 Bde. Carbondale/Edwardsville (zitiert als LW mit Bandangabe).

Fraser, Nancy 2017: „Vom Regen des progressiven Neoliberalismus in die Traufe des reaktionären Populismus“. In: Geiselberger, Heinrich (Hrsg.): Die große Regression. Eine internationale Debatte über die geistige Situation der Zeit. Berlin.

Hacke, Jens 2006: Philosophie der Bürgerlichkeit. Die liberalkonservative Begründung der Bundesrepublik. Göttingen.

Iversen, Torben/Soskice, David 2019: Democracy and Prosperity. Reinventing Capitalism in a Turbulent Century. Oxford.

Jörke, Dirk 2020: „Deweys demokratischer Sozialismus“. In: Brunkhorst, Hauke et al. (Hrsg.): Pragmatistische Sozialtheorie (i.E.).

Katz, Richard S./Mair, Peter 1995: „Changing Models of Party Organization and Party Democracy. The Emergence of the Cartel Party“. In: Party Politics, 1 (1), S. 5-28.

Kennedy, David M. 1999: Freedom From Fear. The American People in Depression and War 1929-1945. Oxford.

Kotkin, Joel 2020: The Coming of Neo Feudalism. A Warning to the Global Middle Class. New York und London.

Mouffe, Chantal 1999: „Dekonstruktion, Pragmatismus und die Politik der Demokratie“. In: Dies. (Hrsg.): Dekonstruktion und Pragmatismus. Demokratie, Wahrheit und Vernunft. Wien, S. 11-37.

Newsinger, John 2018: Hope Lies in the Proles. George Orwell and the Left. London. 
Norris, Christopher 1984: „Language, Truth and Ideology. Orwell and the Post-War Left“. In: Ders.: Orwell. Views from the Left. London, S. 242-262.

Orwell, George 1982: Der Weg nach Wigan Pier. Zürich.

Orwell, George 1987 [1949]: Nineteen Eighty-Four. London.

Paoli, Guillaume 2017: Die lange Nacht der Metamorphose. Über die Gentrifizierung der Kultur. Berlin.

Piketty, Thomas 2020: Kapital und Ideologie. München.

Posner, Richard A. 2001: Public Intellectuals. A Study of Decline. Cambridge.

Reckwitz, Andres 2018: Die Gesellschaft der Singularitäten. Zum Strukturwandel der Moderne. Bonn.

Rorty, Richard 1982: „Dewey’s Metaphysics“. In: Ders.: Consequences of Pragmatism. Essays, 1972-1980. Minneapolis, S. 72-89.

Rorty, Richard 1987: Der Spiegel der Natur. Eine Kritik der Philosophie. Frankfurt.

Rorty, Richard 1991: „Postmodernist bourgeois liberalism“. In: Ders.: Objectivity, Relativism and Truth. New York, S. 175-196.

Rorty, Richard 1999a: Philosophy and Social Hope. London.

Rorty, Richard 1999b: Stolz auf unser Land. Die amerikanische Linke und der Patriotismus. Frankfurt.

Rorty, Richard 1999c: „Bemerkungen zu Dekonstruktion und Pragmatismus“. In: Mouffe, Chantal (Hrsg.): Dekonstruktion und Pragmatismus. Demokratie, Wahrheit und Vernunft. Wien, S. 37-48.

Rorty, Richard 1999d: „Antwort auf Ernesto Laclau“. In: Mouffe, Chantal (Hrsg.): Dekonstruktion und Pragmatismus. Demokratie, Wahrheit und Vernunft. Wien, S. 155-170.

Rorty, Richard 2000a: „Dewey zwischen Hegel und Darwin“. In: Joas, Hans (Hrsg.): Philosophie der Demokratie. Beiträge zum Werk von John Dewey. Frankfurt, S. 20-43.

Rorty, Richard 2000b: „Die Intellektuellen und die Armen“. In: Ders.: Die Schönheit, die Erhabenheit und die Gemeinschaft der Philosophen. Frankfurt, S. 57-87.

Rorty, Richard 2002: Against Bosses, Against Oligarchies. A Conversation with Richard Rorty. Chicago.

Rorty, Richard 2010: „Intellectual Autobiography of Richard Rorty“. In: Auxier, Randalle E./ Hahn, Lewis Edwin (Hrsg.): The Philosophy of Richard Rorty. Chicago, S. 3-24.

Rorty, Richard 2012 [1989]: Kontingenz, Ironie und Solidarität. Frankfurt.

Westbrook, Robert B. (1991): John Dewey and American Democracy. Ithaca. 
